Research Article

Open Access

\title{
Etiologic role of Geotrichum candidum in Oral lesions of an Immunocompromised Patient
}

\author{
Pratibha Dave ${ }^{1}$ and Mahendra Pal ${ }^{2 *}$ \\ ${ }^{\prime}$ Welfare Hospital and Research Center, Bharauch-392001, Gujarat, India \\ ${ }^{2}$ Narayan Consultancy of Veterinary Public Health and Microbiology, 4 Aangan - I, Jagnath Ganesh Dairy Road, Anand-388001, \\ Gujarat, India
}

\section{Article Info}

*Corresponding author:
Mahendra Pal
Narayan Consultancy of Veterinary Public
Health and Microbiology
4 Aangan - I, Jagnath Ganesh Dairy Road
Anand-388001, Gujarat, India
E-mail: palmahendra2@gmail.com

Received: June 20, 2017

Accepted: July 6, 2017

Published: July 13, 2017

Citation: Dave P, Pal M. Etiologic role of Geotrichum candidum in Oral lesions of an Immunocompromised Patient. Madridge J Vaccines. 2017; 1(1): 24-26.

doi: 10.18689/mjv-1000106

\section{Copyright: @ 2017 The Author(s). This work is licensed under a Creative Commons Attribution 4.0 International License, which permits unrestricted use, distribution, and reproduction in any medium, provided the original work is properly cited.}

Published by Madridge Publishers

\begin{abstract}
In recent years, several opportunistic fungi have emerged as an important cause of morbidity and mortality in humans and also in animals. In this paper, we describe the etiologic role of Geotrichum candidum, an opportunistic pathogen, in oral lesions of a 34-years-male immunocompromised patient from Gujarat, India. The scrapings from oral lesions in wet mount preparation of $\mathrm{KOH}$ and Narayan stain showed many elongated rectangular arthrospores morphologically simulating to G. candidum. Similar morphology was observed in cytological smears of oral lesions by Gram technique. The fungus was easily isolated from clinical specimens on Pal sunflower seed medium. Treatment regime included topical application of $1 \%$ solution of gentian violet on the lesions and oral administration of fluconazole. It is emphasized that Narayan stain and Pal sunflower seed medium should be widely employed in Microbiology and Public Health laboratories for the study of fungi, which are implicated in various clinical disorders of humans and also animals. It is advised that immunocompromised patients with oral ulcers should be investigated for fungal infections by employing standard mycological techniques.
\end{abstract}

Keywords: Geotrichum candidum; Immunocompromised patient; Pal sunflower seed medium; Narayan stain.

\section{Introduction}

In recent decades, there is a dramatic increase in the incidence of opportunistic fungal infections as a result of an upsurge in the numbers of immunocompromised patients worldwide [1]. Opportunistic fungal infections of oral cavity are frequently diagnosed in immunocompromised patients and rarely in immunocompetent hosts [2] [3]. Majority of opportunistic oral mucosal fungal infections are attributed to Candida albicans and Aspergillus fumigatus [3]. However, other fungi such as Cryptococcus, Rhodotorula, Geotrichum, Saccharomyces, Fusarium, and Mucor are rarely implicated as opportunistic pathogen in oral cavity [3]. Among several opportunistic fungi, Geotricum candidum is an emerging opportunistic pathogen, which can produce disease in humans as well as in animals [4]. The credit goes to Link who first time isolated Geotrichum candidum from a patient in 1809 [1]. Later, the role of this pathogen in humans and animals is reported from many countries of the world including India [5] [6] [7] [8] [9]. The infection occurs in both sexes and all age groups [4]. The fungus is widely distributed in environment and is recovered from the soil, water, air, vegetables, fruits, milk, silage, plants, besides digestive tract in humans and other animals [10] [4]. Pulmonary involvement due to $G$. candidum is frequently reported but oral, bronchial, vaginal, cutaneous and alimentary infections have also been described [11] [4]. Clinical 
manifestation of oral mycoses includes pseudomembranes, erythema, oedema, ulcers, pustules, abscesses, and extensive tissue necrosis involving bone [3]. Direct microscopy of clinical specimen in $\mathrm{KOH}$, Narayan stain, cytology of Gram stained smear and isolation of pathogen in pure and luxuriant still remains the gold standard for establishing an unequivocal diagnosis of mycotic diseases [1]. A plethora of antifungal drugs such as clotrimazole, miconazole, amphotericin B, fluocytosine, ketoconazole, fluconazole, itraconazole, posaconazle, and voriconazole are tried in the management of fungal diseases [2] [1] [12]. Early diagnosis and prompt therapy is highly imperative in immunocompromised patients to prevent the complications. The paucity of information on the role of Geotrichum candidum in oral lesions of patients, prompted the authors to put on record a case of oral ulcers due to G. candidum from Bharauch, Gujarat, India. In addition, we also report the efficacy of Pal sunflower seed medium for the isolation of $G$. candidum from oral scrapings.

\section{Materials and Methods}

The scrapings collected on two occasions (14 September 2016 and 16 September 2016) aseptically by the ENT surgeon from oral ulcers on the tongue of a 34 -year-male immunocompromised patient constituted the material for this investigation. The patient, a taxi driver, was living in a nearby village of Bharauch, Gujarat, India and was diabetic and HIV positive. He complained low grade fever, persistent cough and weight loss besides lesions in mouth. Direct microscopy of scrapings from tongue lesions was done in wet mount preparation in $10 \%$ potassium hydroxide and Narayan stain [13] to demonstrate fungal elements. Gram stain was employed for cytological examination of smears prepared from oral ulcers of patient. The clinical specimens were inoculated on Sabouraud dextrose agar with chloramphenicol, nutrient agar, blood agar and Pal sunflower seed medium for microbial growth. The incubation temperature for former three media was $37^{\circ} \mathrm{C}$, whereas Pal sunflower seed was kept at $30^{\circ} \mathrm{C}$. The later medium contained $45.0 \mathrm{~g}$ of pulverized seed of sunflower, $20.0 \mathrm{~g}$ agar, $500.0 \mathrm{mg}$ of chloramphenicol [14]. Subculture of suspected colonies was done on APRM medium (Anubha,Pratibha,Raj, Mahendra) for further confirmation. This medium contained $2.0 \mathrm{~g}$ of dried marigold flowers, $2.0 \mathrm{~g}$ agar, $50 \mathrm{mg}$ chloramphenicol and $100 \mathrm{ml}$ distilled water [15]. Cycloheximide should not be incorporated in cultural medium as it inhibits the growth of Geotrichum. Detailed microscopic morphology of cultures grown on APRM medium was studied in Narayan stain, which contained $4 \mathrm{ml}$ of glycerin, $6.0 \mathrm{ml}$ of dimethyl sulfoxide (DMSO) and $.05 \mathrm{ml}$ of $3 \%$ solution of methylene blue [13]. The treatment was done with local application of $1 \%$ gentian violet lotion and oral administration of fluconazole tablets (100 mg twice daily) for about 4- 5 weeks. The patient was advised to report clinical response of therapy to the surgeon.

\section{Results}

Detailed clinical examination of patient showed white patches over dorsal surface of tongue with irregular and inflamed margins. The tongue surfaces in between revealed fissures. In addition, patient experienced burning pain and impaired swallowing. The microscopic examination of clinical materials in $\mathrm{KOH}$ mount and Narayan stain mount showed multiple branched septate, hyaline hyphae and rectangular arthroconidia. Cytology of smears from oral lesions in Gram stain revealed numerous elongated, rectangular arthroconidia and septate hyphae morphologically indistinguishable from Geotrichum candidum. There was no growth of bacteria on nutrient agar and blood agar. However, on both mycological media, colonies were fast growing, dry, flat, white to cream with no reverse pigment. Subculture of suspected colonies on APRM medium in Narayan stain revealed hyaline septate branched hyphae and smooth arthroconidia. There was no formation of Germ tube, urease test and nitrate test were negative, and glucose assimilation test was positive. Colonies on Pal sunflower seed failed to show brown pigment. The cultural, morphological and biochemical characteristics confirmed the identity of isolates as G. candidum [1]. Patient showed gradual clinical improvement with the prescribed drugs, and did not report any side effects of the medication to the Surgeon.

\section{Discussion}

Geotrichum candidum, the principal causative agent of geotrchosis, is an opportunistic pathogen of global distribution. The fungus has gained clinical significance in immunocompromised hosts due some underlying diseases such as HIV/AIDS, diabetes mellitus, neoplasm, besides renal transplants [5] [4] [3]. Geotrichum candidum can cause life threatening infection in immunocompromised patients. In the present case, the clinical observations, mycological findings and therapeutic response clearly indicated that our patient was suffering with oral mycosis due to Geotrichum candidum. The results of our investigation go parallel with other the findings of other researchers who reported $G$. candidum infection of oral cavity from several countries [5] [8] [9]. As prognosis of disseminated disease in immunocompromised patient is grave, prompt treatment with antifungal drugs is pertinent to save the life of patient. We immediately started the therapy and our patient responded well with topical application of gentian violet and oral administration of fluconazole.

The isolation of $\mathrm{G}$. candidum from oral specimens on Pal sunflower seed medium is an interesting observation and needs further studies to evaluate its efficacy to isolate this emerging opportunistic fungus. Pal sunflower seed medium was developed by Pal in 1980 for the rapid isolation and identification of Cryptococcus neoformans from clinical and environmental materials [1]. It is, therefore, advised that this medium being very cheap than other mycological media, can be successfully tried for the isolation of $G$. candidum from a variety of sources. 
It is pertinent to differentiate $G$. candium infection from other fungal diseases such as candidiasis, aspergillosis, and zygomycosis [9]. The correct early diagnosis and prompt therapy with specific antifungal drugs is imperative to prevent the morbidity of disease [1]. As Dental Clinician plays a pivotal role in the diagnosis and management of oral fungal diseases, it is, therefore, pertinent to create awareness about the role of opportunistic fungi growing in fungal infections of oral cavity. The routine application of Pal sunflower seed medium for isolation and Narayan stain for morphological identification of fungi is recommended in primary health centers located in rural areas of poor resource countries, which hardly have good laboratory facilities for the diagnosis of mycotic diseases. It is recommended that growing role of G.candidum in various clinical disorders of humans and animals should be further investigated.

\section{Acknowledgements}

We thank to Ear, Nose and Throat Department of Welfare Hospital and Research Center, Bharauch, Gujarat, India, for the submission of clinical samples, and also to the staff of Laboratory for technical assistance. The cooperation of patient during collection of samples is also thankfully acknowledged. The help of Anubha in computer is highly appreciated.

\section{References}

1. Pal M. Veterinary and Medical Mycology. $1^{\text {st }}$ Ed. Indian Council of Agricultural Research, New Delhi, India 2007.

2. Pal M, Desai MT. Oral ulcers in an immunocompetent patient due to Candida albicans. Antiseptic. 1998; 95: 50-51.
3. Deepa AG, Nair BJ, Sivkumar TT, Joseph AP. Uncommon opportunistic fungal infections of oral cavity: A review. J Oral Maxillofac Pathol. 2014; 18: 235-43. doi: 10.4103/0973-029X.140765

4. Pal M, Sejra S, Sejra A, Tesfaye S. Geotrichosis: An opportunistic mycosis of humans and animals. Int. J. Livest. Res. 2013; 3(2): 38-44.

5. Heinic GS, Greenspan D, MacPhail LA, Greenspan JS. Oral Geotrichum candidum infection associated with HIV patient, a case report. Oral Surg Oral Med Oral Pathol. 1992; 73: 726-28.

6. Pal M, Lee CW, Matsusaka N. Occurrence of Geotrichum candidum in two cases of canine dermatitis. J Wet Clin. 2002; 19: 433-435.

7. Pal M. Role of Geotrichum candidum in canine oral ulcers. Revista Iberoamericana de Micologia. 2005; 22(3): 183.

8. Hattori $\mathrm{H}$, Inoue $\mathrm{C}$, Tomita $\mathrm{Y}, \mathrm{Kanbe} \mathrm{T}$. A case of oral geotrichosis caused by Geotrichum candidum in an old patient. Jpn J Infect Dis. 2007; 60: 300-301.

9. Bonifaz A, Gonzalez DZ, Macias B, Parades-Farrera F, Hernandez MA, Arazia J. Oral geotrichosis: Report of 12 cases. J Oral Sci. 2010; 52(3): 477-483.

10. Samaranayake LP, Keung W, Jin L. Oral mucosal fungal infections. Periodontol . 2009; 49: 39-59. doi: 10.1111/j.1600-0757.2008.00291.x

11. Krishnan PA. Fungal infections of the oral mucosa. Indian J Dent Res. 2012; 23: 650-659.

12. Chander J. Textbook of Medical Mycology. $3^{\text {rd }}$ Ed. Mehta Publishers, New Delhi, India 2009.

13. Pal M. Efficacy of "Narayan" stain for morphological studies of mould, yeasts and algae. Revista Iberoamericana de Micologia. 2004; 21: 219.

14. Pal M. Use of Pal's sunflower medium for an early diagnosis of cryptococcosis. Antiseptic. 1997; 95(5): 175.

15. Dave $\mathrm{P}$, Mahendra R, Pal M. Etiologic significance of Aspergillus terreus in primary cutaneous mycosis of an agricultural worker. Mol. Microb. Res. 2015; 5(2): 1-4. doi: 10.5376/mmr.2015.05.0002 